\title{
Effect of red algal bloom on growth and production of carps
}

\author{
Ram Bhajan Mandal", Sunila Rai**, Madhav Kumar Shrestha***, Dilip Kumar \\ Jha**** and Narayan Prasad Pandit*****
}

\author{
Agriculture and Forestry University, Rampur, Chitwan, Nepal \\ *E-mail: rbmandal2008@gmail.com \\ **E-mail: sunilarai10@gmail.com \\ ***E-mail: madhavshrestha1954@gmail.com \\ ****E-mail: dkjha.ait@gmail.com \\ *****E-mail: panditnp@hotmail.com
}

\begin{abstract}
s
An experiment was carried to assess the effect of red algal bloom on growth and production of carp, water quality and profit from carp for 120 days at Aquaculture Farm of Agriculture and Forestry University, Chitwan. The experiment included two treatments: carp polyculture in non-red pond and carp polyculture in red pond with algal bloom each with three replicates. Carp fingerlings were stocked at $1 \mathrm{fish} / \mathrm{m}^{2}$ and fed with pellet containing $24 \% \mathrm{CP}$ at $3 \%$ body weight. Net yield of rohu was found significantly higher $(\mathrm{p}<0.05)$ in non-red ponds $\left(0.38 \pm 0.01 \mathrm{t} \mathrm{ha}^{-1}\right)$ than red ponds $\left(0.24 \pm 0.05 \mathrm{t} \mathrm{ha}^{-1}\right)$. Survival of rohu $(84.9 \pm 1.4 \%)$, bighead $(95.2 \pm 2.0 \%)$ and mrigal $(88.1 \pm 14.4 \%)$ were also significantly higher $(\mathrm{p}<0.05)$ in non-red ponds than red ponds. Red algal bloom affected DO, nitrate and chlorophyll-a, nitrite, total nitrogen, total phosphorus, total dissolved solids and conductivity. However, overall carp production and profit from carp remained unaffected.
\end{abstract}

Key words: Euglenophytes, Red pond, Carp polyculture, Water quality

\footnotetext{
DOI: http://dx.doi.org/10.3126/on.v16i1.22122

Manuscript details: Received: 24.04.2018 / Accepted: 16.07.2018

Citation: Mandal, R.B., S. Rai, M.K. Shrestha, D.K. Jha and N.P. Pandit 2018. Effect of red algal bloom on growth and production of carp, Our Nature 16(1): 48-54. DOI: http://dx.doi.org/ 10.3126/on.v16i1.22122

Copyright: @ Mandal, Rai, Shrestha, Jha and Pandit 2018. Creative Commons Attribution - Non Commercial 4.0 International License.
}

\section{Introduction}

Red algal bloom is the common phenomenon in warmer shallow and eutrophic water bodies (Rahman and Khan, 2007; Rodgers, 2008). Eutrophic condition induces toxic and noxious phytoplankton bloom including euglenophytes which causes red blooming in pond water (Ohio EPA, 2013). It was found that Euglenophytes dominated by Euglena sanguinea causes red blooming in ponds (Ohio EPA, 2013). It was found that Euglenophytes and Euglena sanguinea dominates phytoplankton community in red bloom ponds (Mandal et al., 2016). Euglena assemblages are known to be widely distributed in higher eutrophic shallow ponds at elevated temperature (Wild et al., 1995). In red ponds, film or scum at the surface gives unpleasant look, shades the lower waters, inhibit photosynthesis, deplete dissolved oxygen, brings behavioral changes in fish and sometimes results fish mortality too (Rehman, 1998; Zimba et al., 2004 ; 2010) due to euglenoid toxin functioning as a neurotoxin (Costa and Garrido, 2004; Costa, 2014). When population of Euglena sanguinea increases dramatically, it causes oxygen depletions killing fish, shellfish and other aquatic organisms (Lopez et al., 2008; Zimba et al., 2004; 2010; Boyd and Tucker, 2014).

In Nepal, farmers generally believe that red bloom has adverse effects on fish farming such as low fish production, oxygen depletion and fish mortality; however, no scientific research 
has been done yet to prove it. Therefore, present research was to assess effect of red algal bloom on growth and production of carp in pond.

\section{Materials and methods}

The experiment was carried out in six earthen ponds at the Aquaculture Farm, Agricultural and Forestry University (AFU), Chitwan, Nepal. The experiment included two treatments, I) carp polyculture in non-red pond and II) carp polyculture in red pond with algal bloom with three replications. The experiment was carried out for 120 days from 18 April to 17 August 2017. Prior to stocking, all ponds were completely drained and filled with canal water to $1.2 \mathrm{~m}$. Red ponds were fertilized with goat manure at a rate of $3 \mathrm{~kg} 100 \mathrm{~m}^{-2}$ of the pond area on dry weight basis biweekly. Non-red ponds

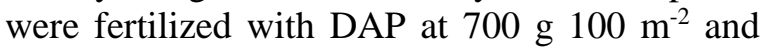

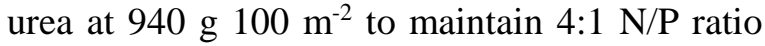
biweekly. In both treatment ponds, carp was stocked at a rate of 1 fish $\mathrm{m}^{-2}$. The fish were silver carp (Hypophthalmychthys molitrix), common carp (Cyprinus carpio), rohu (Labeo rohita), mrigal (Cirrhinus mrigala), bighead carp (Aristichthys nobilis) and grass carp (Ctenopharingodon idella) at 3.5: 2.5: 1.5: 1:1:0.5 ratio, respectively. Floating pellet manufactured by Him feed company (24\% CP) was fed at $3 \%$ body weight per day to carp except grass carp. Grass carp was fed with grass on wet weight basis of $50 \%$ body weight. Feed proximate analysis showed that pellet contained 91.5\% dry matter, $24.0 \%$ crude protein, $5.5 \%$ crude fiber, $5.6 \%$ ether extract, $5.3 \%$ total ash and $51.0 \%$ nitrogen free extract. Fish growth was assessed monthly by sampling $10 \%$ population of each species to adjust feed ration.

Water quality parameters such as temperature, dissolved oxygen (DO), $\mathrm{pH}$, oxidation reduction potential (ORP), conductivity, total dissolved solid (TDS) were analyzed in situ using HI-98194 Multiparameter whereas soluble reactive phosphorus (SRP), nitrate, nitrite, ammonia nitrogen $\left(\mathrm{NH}_{3}-\mathrm{N}\right)$ were analyzed using HI-83203-02 Multi parameter bench photometer biweekly. Total phosphorus, total nitrogen and chlorophyll-a was analyzed following standard methods (APHA, 2012). Plankton samples were taken at 06:00-07:00 hours by using column sampler and preserved in 5\% formaldehyde solution. Phytoplanktons specially the euglenoids were identified to generic level by using Prescott (1951), Rai and Rai (2007), and Guiry and Guiry (2016). The
Zooplankton were identified to generic level by using Edmondson (1959), Pennak (1978), Reddy (1994) and Dhanapathi (2000). Planktons were counted using Sedgwick-Rafter (S-R) cells and quantified following APHA (2012).

No of species $=\mathrm{C} \times 1000 \mathrm{~mm}^{3} / \mathrm{L} \times \mathrm{D} \times \mathrm{W} \times$ $\mathrm{S}$

Where, $\mathrm{C}=$ Number of organisms counted, $\mathrm{L}=$ Length of each stripe $(\mathrm{mm}), \mathrm{D}=$ Depth of each stripe $(\mathrm{mm}), \mathrm{W}=$ Width of each stripe $(\mathrm{mm})$, and $\mathrm{S}=$ Number of stripes.

Gross margin analysis was done to determine gross margin of each treatment (Shang, 1990). The analysis was based on market prices in Nepal for harvested fish and all other items. Market prices of harvested carp were $270 \mathrm{NPR} \mathrm{kg}^{-1}$. Market prices of carp fingerlings were $2 \mathrm{NPR}$ piece $^{-1}$. The market

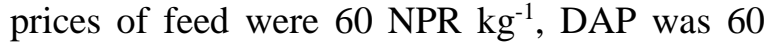

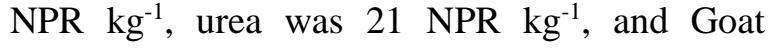
manure was free of cost.

Data were analyzed statistically by using SPSS (version 16). Independent t-test with twotailed test was done to compare means between two treatments. Alpha level was set at 5\%. All means were given with \pm 1 standard deviation (SD).

\section{Results and discussion Growth and yield of fish}

The initial weight, harvest weight, daily weight gain, and survival of carp are presented in Table 1 while combined initial weight $\left(\mathrm{kg} \mathrm{ha}^{-1}\right)$, final weight $\left(\mathrm{kg} \mathrm{ha}^{-1}\right)$, net fish yield $\left(\mathrm{t} \mathrm{ha}{ }^{-1}\right)$ and extrapolated net fish yield $\left(\mathrm{t} \mathrm{ha}^{-1} \mathrm{year}^{-1}\right)$ of carp are presented in Table 2 .

Combined initial and final weight, net fish yield and extrapolated net fish yield and survival were not significantly different $(\mathrm{P}>0.05)$ between non-red and red ponds. Net fish yield of rohu was significantly higher $(\mathrm{P}<0.05)$ in nonred $\left(0.38 \pm 0.01 \mathrm{t} \mathrm{ha}^{-1}\right)$ than red pond $(0.24 \pm 0.05 \mathrm{t}$ $\left.\mathrm{ha}^{-1}\right)$. Survival of rohu $(84.9 \pm 1.4 \%)$ bighead $(95.2 \pm 2.0 \%)$ and mrigal $(88.1 \pm 14.4 \%)$ were also significantly higher in non-red ponds than red ponds of rohu $(61.6 \pm 19.5 \%)$ bighead $(86.0 \pm 3.2$ $\%)$ and mrigal (59.7 $\pm 24.8 \%)$.

Fish growth in semi-intensive system depends on a variety of factors out of which the most important are environmental factors, nutrients and plankton population. In the present study, the growth of all carp species except rohu and combined production did not differ between non-red and red ponds indicating red algal bloom does not affect growth and production of carp 
Table 1. Stocking weight, harvest weight, daily weight gain, net fish yield and survival of carp (Mean \pm SD) in non-red and red ponds with algal bloom.

\begin{tabular}{|c|c|c|}
\hline \multirow{2}{*}{$\begin{array}{l}\text { Parameter } \\
\text { Silver carp } \\
\end{array}$} & \multicolumn{2}{|l|}{ Treatments } \\
\hline & Non-Red & Red Pond \\
\hline Initial mean weight $\left(\mathrm{g}_{\text {fish }}{ }^{-1}\right)$ & $19.0 \pm 2.0^{\mathrm{a}}$ & $17.8 \pm 1.5^{\mathrm{a}}$ \\
\hline Initial total weight $\left(\mathrm{kg} \mathrm{ha}^{-1}\right)$ & $66.5 \pm 7.0^{\mathrm{a}}$ & $62.4 \pm 9.6^{\mathrm{a}}$ \\
\hline Final mean weight $\left(\mathrm{g} \mathrm{fish}^{-1}\right)$ & $289.3 \pm 28.2^{\mathrm{a}}$ & $253.6 \pm 90.0^{\mathrm{a}}$ \\
\hline Final total weight $\left(\mathrm{kg} \mathrm{ha}^{-1}\right)$ & $524.9 \pm 90.3^{\mathrm{a}}$ & $398.3 \pm 141.7^{\mathrm{a}}$ \\
\hline Daily weight gain $\left(\mathrm{g} \mathrm{fish}^{-1} \mathrm{day}^{-1}\right)$ & $2.3 \pm 0.2^{\mathrm{a}}$ & $2.0 \pm 0.8^{\mathrm{a}}$ \\
\hline Total weight gain $\left(\mathrm{kg} \mathrm{ha}^{-1}\right)$ & $458.4 \pm 7.0^{\mathrm{a}}$ & $335.9 \pm 7.0^{\mathrm{a}}$ \\
\hline Net fish yield $\left(\mathrm{t} \mathrm{ha}^{-1}\right)$ & $0.46 \pm 0.09^{\mathrm{a}}$ & $0.34 \pm 0.1^{\mathrm{a}}$ \\
\hline Extrapolated net fish yield $\left(\mathrm{t} \mathrm{ha}^{-1} \mathrm{yr}^{-1}\right)$ & $1.38 \pm 0.3^{\mathrm{a}}$ & $1.1 \pm 0.4^{\mathrm{a}}$ \\
\hline Survival $(\%)$ & $52.7 \pm 13.8^{\mathrm{a}}$ & $52.3 \pm 36.7^{\mathrm{a}}$ \\
\hline \multicolumn{3}{|l|}{ Bighead carp } \\
\hline Initial mean weight $\left(\mathrm{g} \mathrm{fish}^{-1}\right)$ & $40.7 \pm 3.1^{\mathrm{a}}$ & $41.7 \pm 1.5^{\mathrm{a}}$ \\
\hline Initial total weight $\left(\mathrm{kg} \mathrm{ha}^{-1}\right)$ & $40.7 \pm 3.6 .0^{\mathrm{a}}$ & $41.7 \pm 1.5^{\mathrm{a}}$ \\
\hline Final mean weight $\left(\mathrm{g} \mathrm{fish}^{-1}\right)$ & $349.3 \pm 44.4^{\mathrm{a}}$ & $378.8 \pm 84.3^{\mathrm{a}}$ \\
\hline Final total weight $\left(\mathrm{kg} \mathrm{ha}^{-1}\right)$ & $332.9 \pm 48.2^{\mathrm{a}}$ & $448.4 \pm 210.9^{a}$ \\
\hline Daily weight gain $\left(\mathrm{g} \mathrm{fish}^{-1} \mathrm{day}^{-1}\right)$ & $2.6 \pm 0.8^{\mathrm{a}}$ & $2.8 \pm 0.7^{\mathrm{a}}$ \\
\hline Total weight gain $\left(\mathrm{kg} \mathrm{ha}^{-1}\right)$ & $292.2 \pm 51.2^{\mathrm{a}}$ & $406.8 \pm 209.3^{\mathrm{a}}$ \\
\hline Net fish yield $\left(\mathrm{t} \mathrm{ha}^{-1}\right)$ & $0.29 \pm 0.05^{\mathrm{a}}$ & $0.41 \pm 0.2^{\mathrm{a}}$ \\
\hline Extrapolated net fish yield $\left(\mathrm{t} \mathrm{ha}^{-1} \mathrm{yr}^{-1}\right)$ & $0.88 \pm 0.2^{\mathrm{a}}$ & $1.22 \pm 0.6^{\mathrm{a}}$ \\
\hline Survival $(\%)$ & $95.2 \pm 2.0^{\mathrm{a}}$ & $86.0 \pm 3.2^{\mathrm{b}}$ \\
\hline \multicolumn{3}{|l|}{ Common carp } \\
\hline Initial mean weight $\left(\mathrm{g} \mathrm{fish}^{-1}\right)$ & $18.0 \pm 1.0^{\mathrm{a}}$ & $19.2 \pm 0.8^{\mathrm{a}}$ \\
\hline Initial total weight $\left(\mathrm{kg} \mathrm{ha}^{-1}\right)$ & $45.0 \pm 2.5^{\mathrm{a}}$ & $47.9 \pm 1.9^{\mathrm{a}}$ \\
\hline Final mean weight $\left(\mathrm{g} \mathrm{fish}^{-1}\right)$ & $283.1 \pm 4.2^{\mathrm{a}}$ & $292.1 \pm 83.2^{\mathrm{a}}$ \\
\hline Final total weight $\left(\mathrm{kg} \mathrm{ha}^{-1}\right)$ & $632.4 \pm 67.4^{\mathrm{a}}$ & $622.0 \pm 151.2^{\mathrm{a}}$ \\
\hline Daily weight gain $\left(\mathrm{g} \mathrm{fish}^{-1} \mathrm{day}^{-1}\right)$ & $2.2 \pm 0.1^{\mathrm{a}}$ & $2.3 \pm 0.7^{\mathrm{a}}$ \\
\hline Total weight gain $\left(\mathrm{kg} \mathrm{ha}^{-1}\right)$ & $587.4 \pm 69.9^{a}$ & $574.1 \pm 151.3^{\mathrm{a}}$ \\
\hline Net fish yield $\left(\mathrm{t} \mathrm{ha}^{-1}\right)$ & $0.59 \pm 0.07^{\mathrm{a}}$ & $0.57 \pm 0.2^{\mathrm{a}}$ \\
\hline Extrapolated net fish yield $\left(\mathrm{t} \mathrm{ha}^{-1} \mathrm{yr}^{-1}\right)$ & $1.76 \pm 0.2^{\mathrm{a}}$ & $1.72 \pm 0.4^{\mathrm{a}}$ \\
\hline Survival $(\%)$ & $89.5 \pm 10.7^{\mathrm{a}}$ & $86.7 \pm 21.0^{\mathrm{a}}$ \\
\hline \multicolumn{3}{|l|}{ Rohu } \\
\hline Initial mean weight $\left(\mathrm{g} \mathrm{fish}^{-1}\right)$ & $19.3 \pm 4.2^{\mathrm{a}}$ & $16.7 \pm 3.1^{\mathrm{a}}$ \\
\hline Initial total weight $\left(\mathrm{kg} \mathrm{ha}^{-1}\right)$ & $29.0 \pm 6.2^{\mathrm{a}}$ & $25.0 \pm 4.9^{\mathrm{a}}$ \\
\hline Final mean weight $\left(\mathrm{g} \mathrm{fish}^{-1}\right)$ & $324.4 \pm 6.0^{\mathrm{a}}$ & $303.6 \pm 60.7^{\mathrm{a}}$ \\
\hline Final total weight $\left(\mathrm{kg} \mathrm{ha}^{-1}\right)$ & $413.1 \pm 0.9^{\mathrm{a}}$ & $269.1 \pm 47.5^{\mathrm{b}}$ \\
\hline Daily weight gain $\left(\mathrm{g} \mathrm{fish}^{-1} \mathrm{day}^{-1}\right)$ & $2.5 \pm 0.1^{\mathrm{a}}$ & $2.4 \pm 0.5^{\mathrm{a}}$ \\
\hline Total weight gain $\left(\mathrm{kg} \mathrm{ha}^{-1}\right)$ & $384.1 \pm 6.9^{\mathrm{a}}$ & $244.1 \pm 45.4^{\mathrm{b}}$ \\
\hline Net fish yield $\left(\mathrm{t} \mathrm{ha}^{-1}\right)$ & $0.38 \pm 0.01^{\mathrm{a}}$ & $0.24 \pm 0.05^{\mathrm{b}}$ \\
\hline Extrapolated net fish yield $\left(\mathrm{t} \mathrm{ha}^{-1} \mathrm{yr}^{-1}\right)$ & $1.15 \pm 0.02^{\mathrm{a}}$ & $0.73 \pm 0.1^{\mathrm{b}}$ \\
\hline Survival $(\%)$ & $84.9 \pm 1.4^{\mathrm{a}}$ & $61.6 \pm 19.5^{\mathrm{b}}$ \\
\hline \multicolumn{3}{|l|}{ Mrigal } \\
\hline Initial mean weight $\left(\mathrm{g} \mathrm{fish}^{-1}\right)$ & $18.7 \pm 1.5^{\mathrm{a}}$ & $16.3 \pm 1.1^{\mathrm{a}}$ \\
\hline
\end{tabular}




\begin{tabular}{|c|c|c|}
\hline Initial total weight $\left(\mathrm{kg} \mathrm{ha}^{-1}\right)$ & $18.7 \pm 1.5^{\mathrm{a}}$ & $16.4 \pm 1.2^{\mathrm{a}}$ \\
\hline Final mean weight $\left(\mathrm{g} \mathrm{fish}^{-1}\right)$ & $231.3 \pm 12.9^{\mathrm{a}}$ & $197.0 \pm 45.2^{\mathrm{a}}$ \\
\hline Final total weight $\left(\mathrm{kg} \mathrm{ha}^{-1}\right)$ & $250.8 \pm 74.5^{\mathrm{a}}$ & $125.1 \pm 82.1^{\mathrm{a}}$ \\
\hline Daily weight gain $\left(\mathrm{g} \mathrm{fish}^{-1} \mathrm{day}^{-1}\right)$ & $1.8 \pm 0.1^{\mathrm{a}}$ & $1.5 \pm 0.4^{\mathrm{a}}$ \\
\hline Total weight gain $\left(\mathrm{kg} \mathrm{ha}^{-1}\right)$ & $231.6 \pm 75.6^{\mathrm{a}}$ & $108.7 \pm 82.9^{\mathrm{a}}$ \\
\hline Net fish yield $\left(\mathrm{t} \mathrm{ha}^{-1}\right)$ & $0.23 \pm 0.1^{\mathrm{a}}$ & $0.11 \pm 0.1^{\mathrm{a}}$ \\
\hline Extrapolated net fish yield $\left(\mathrm{t} \mathrm{ha}^{-1} \mathrm{yr}^{-1}\right)$ & $0.69 \pm 0.2^{\mathrm{a}}$ & $0.33 \pm 0.3^{\mathrm{a}}$ \\
\hline Survival $(\%)$ & $88.1 \pm 14.4^{\mathrm{a}}$ & $59.7 \pm 24.8^{\mathrm{b}}$ \\
\hline \multicolumn{3}{|l|}{ Grass carp } \\
\hline Initial mean weight $\left(\mathrm{g} \mathrm{fish}^{-1}\right)$ & $42.2 \pm 5.0^{\mathrm{a}}$ & $37.3 \pm 9.1^{\mathrm{a}}$ \\
\hline Initial total weight $\left(\mathrm{kg} \mathrm{ha}^{-1}\right)$ & $21.0 \pm 2.6^{\mathrm{a}}$ & $18.7 \pm 4.5^{\mathrm{a}}$ \\
\hline Final mean weight $\left(\mathrm{g}_{\text {fish }}{ }^{-1}\right)$ & $419.4 \pm 70.9^{\mathrm{a}}$ & $500.7 \pm 150.5^{\mathrm{a}}$ \\
\hline Final total weight $\left(\mathrm{kg} \mathrm{ha}^{-1}\right)$ & $83.9 \pm 5.4^{\mathrm{a}}$ & $136.5 \pm 65.6^{\mathrm{a}}$ \\
\hline Daily weight gain $\left(\mathrm{g} \mathrm{fish}^{-1} \mathrm{day}^{-1}\right)$ & $3.1 \pm 0.6^{\mathrm{a}}$ & $3.9 \pm 1.2^{\mathrm{a}}$ \\
\hline Total weight gain $\left(\mathrm{kg} \mathrm{ha}^{-1}\right)$ & $62.9 \pm 7.7^{\mathrm{a}}$ & $117.8 \pm 67.7^{\mathrm{a}}$ \\
\hline Net fish yield $\left(\mathrm{t} \mathrm{ha}^{-1}\right)$ & $0.06 \pm 0.01^{\mathrm{a}}$ & $0.12 \pm 0.07^{\mathrm{a}}$ \\
\hline Extrapolated net fish yield $\left(\mathrm{t} \mathrm{ha}^{-1} \mathrm{yr}^{-1}\right)$ & $0.19 \pm 0.02^{\mathrm{a}}$ & $0.35 \pm 0.2^{\mathrm{a}}$ \\
\hline Survival (\%) & $40.5 \pm 4.1^{\mathrm{a}}$ & $60.8 \pm 38.3^{\mathrm{a}}$ \\
\hline
\end{tabular}

Mean values with different superscript letters in the same row are significantly different $(\mathrm{P}<0.05)$.

Table 2. Combined initial total weight $\left(\mathrm{kg} \mathrm{ha}^{-1}\right)$, combined final total weight $\left(\mathrm{kg} \mathrm{ha}^{-1}\right)$, combined net fish yield ( $\mathrm{t}$ $\mathrm{ha}^{-1}$ ), survival of carp and AFCR (Mean \pm SD) in 120 days in non-red and red ponds with algal bloom

\begin{tabular}{|l|l|l|}
\hline \multirow{2}{*}{ Parameter } & Treatments & \multicolumn{1}{l|}{ Red Pond } \\
\cline { 2 - 3 } & Non-Red & $212.0 \pm 12.9^{\mathrm{a}}$ \\
\hline Initial total weight $\left({\left.\mathrm{kg} . \mathrm{ha}^{-1}\right)}^{-1}\right)$ & $220.8 \pm 4.0^{\mathrm{a}}$ & $1999.9 \pm 464.4^{\mathrm{a}}$ \\
\hline Final total weight $\left(\mathrm{kg} \cdot \mathrm{ha}^{-1}\right)$ & $2238.7 \pm 188.4^{\mathrm{a}}$ & $1787.4 \pm 451.8^{\mathrm{a}}$ \\
\hline Net fish yield $\left(\mathrm{kg} \cdot \mathrm{ha}^{-1}\right)$ & $2017.2 \pm 190.2^{\mathrm{a}}$ & $1.8 \pm 0.5^{\mathrm{a}}$ \\
\hline Net fish yield (t.ha-1) & $2.02 \pm 0.2^{\mathrm{a}}$ & $5.4 \pm 1.7^{\mathrm{a}}$ \\
\hline Extrapolated net fish yield $\left(\mathrm{t} \mathrm{ha}^{-1} \mathrm{yr}^{-1}\right)$ & $6.1 \pm 0.6^{\mathrm{a}}$ & $67.8 \pm 8.5^{\mathrm{a}}$ \\
\hline Survival (\%) & $75.2 \pm 5.8^{\mathrm{a}}$ & $1.9 \pm 0.5^{\mathrm{a}}$ \\
\hline AFCR & $1.5 \pm 0.2^{\mathrm{a}}$ & $\mathrm{P}<0.05)$. \\
\hline
\end{tabular}

Mean values with different superscript letters in the same row were significantly different $(\mathrm{P}<0.05)$.

critically. Net fish yield of rohu in non-red pond was significantly higher $\left(0.38 \pm 0.01 \mathrm{t} \mathrm{ha}^{-1}\right)$ than red pond $\left(0.24 \pm 0.05 \mathrm{tha}^{-1}\right)$ in relation to higher survival and weight gain which was probably due to higher DO and chlorophytes (Rai et al. 2010 ) in non-red ponds. Red algal blooms results oxygen deficiency which greatly affect the growth and production of fish (Xavier et al., 1991). Although total fish production in red pond $\left(5.4 \pm 1.7 \mathrm{t} \mathrm{ha}^{-1} \mathrm{yr}^{-1}\right)$ was comparatively lower than non-red pond $\left(6.1 \pm 0.6 \mathrm{tha}^{-1} \mathrm{yr}^{-1}\right)$ but it was still slightly higher than average fish yield of $4.9 \mathrm{t} \mathrm{ha}^{-1} \mathrm{yr}^{-1}$ in Nepal (DoFD, 2016). This indicated that red algal bloom is not disastrous to fish production. Survival of rohu, bighead and mrigal were significantly lower in red ponds probably due to lower DO and higher nitrite (Ciji et al., 2014). Toxic nitrite content increases mortality of fish (Ciji et al., 2014).

\section{Water quality}

Water quality parameters such as $\mathrm{pH}$, temperature, Oxidation reduction potential (ORP), soluble reactive phosphorus (SRP) and $\mathrm{NH}_{3}-\mathrm{N}$ were not significantly different $(\mathrm{P}>0.05)$ between non-red and red ponds. Dissolved oxygen (DO), nitrate and chlorophyll-a were significantly higher $(\mathrm{P}<0.05)$ in non-red ponds than red ponds while nitrite, TP, TN, TDS and conductivity were significantly $(\mathrm{P}<0.05)$ higher in red ponds than non-red ponds (Table 3 ).

Dissolved oxygen was significantly lower in red pond $(1.1 \pm 0.2 \mathrm{mg} / \mathrm{L})$ than non-red pond $(2.8 \pm 0.1 \mathrm{mg} / \mathrm{L})$ which might be due to the bloom 
Table 3. Water quality parameter in non-red ponds and red ponds with algal bloom during experimental period $($ Mean \pm SD).

\begin{tabular}{|c|c|c|}
\hline Parameter & Non-Red & Red pond \\
\hline Temperature $\left({ }^{\circ} \mathrm{C}\right)$ & $29.5 \pm 0.1^{\mathrm{a}}$ & $29.3 \pm 0.01^{\mathrm{a}}$ \\
\hline $\mathrm{pH}$ & $7.6 \pm 0.0(7.6-7.7)$ & $7.4 \pm 0.1(7.3-7.5)$ \\
\hline $\mathrm{DO}\left(\mathrm{mg} . \mathrm{L}^{-1}\right)$ & $2.8 \pm 0.1^{\mathrm{a}}$ & $1.1 \pm 0.2^{\mathrm{b}}$ \\
\hline ORP $(\mathrm{mV})$ & $76.0 \pm 4.1^{\mathrm{a}}$ & $75.0 \pm 2.7^{\mathrm{a}}$ \\
\hline $\mathrm{NH}_{3}-\mathrm{N}\left(\mathrm{mg} . \mathrm{L}^{-1}\right)$ & $0.79 \pm 0.28^{\mathrm{a}}$ & $0.81 \pm 0.06^{\mathrm{a}}$ \\
\hline SRP (mg. L $\left.{ }^{-1}\right)$ & $0.21 \pm 0.05^{\mathrm{a}}$ & $0.22 \pm 0.06^{\mathrm{a}}$ \\
\hline Nitrate (mg. L $\left.{ }^{-1}\right)$ & $0.59 \pm 0.07^{\mathrm{a}}$ & $0.21 \pm 0.09^{\mathrm{b}}$ \\
\hline Nitrite (mg. L $\left.{ }^{-1}\right)$ & $0.04 \pm 0.01^{\mathrm{b}}$ & $0.12 \pm 0.03^{\mathrm{a}}$ \\
\hline Chlorophyll-a (mg. $\left.\mathrm{L}^{-3}\right)$ & $32.8 \pm 2.6^{\mathrm{a}}$ & $18.8 \pm 2.6^{\mathrm{b}}$ \\
\hline TDS (mg. L-1) & $112.2 \pm 13.7^{\mathrm{b}}$ & $141.9 \pm 10.1^{\mathrm{a}}$ \\
\hline Conductivity $\left(\mu \mathrm{S} . \mathrm{cm}^{-1}\right)$ & $224.2 \pm 23.8^{\mathrm{b}}$ & $281.6 \pm 19.8^{a}$ \\
\hline $\mathrm{TN}\left(\mathrm{mg} . \mathrm{L}^{-1}\right)$ & $2.8 \pm 0.4^{\mathrm{b}}$ & $4.4 \pm 0.4^{\mathrm{a}}$ \\
\hline $\mathrm{TP}\left(\mathrm{mg} . \mathrm{L}^{-1}\right)$ & $0.45 \pm 0.01^{\mathrm{b}}$ & $0.53 \pm 0.05^{\mathrm{a}}$ \\
\hline
\end{tabular}

Mean values with different superscript letters in the same row are significantly different $(\mathrm{P}<0.05)$.

Table 4. Abundance of phytoplankton and zooplankton $\left(10^{3}\right.$ cells. $\left.\mathrm{L}^{-1}\right)$ in non-red ponds and red ponds with algal bloom during experimental period (Mean $\pm \mathrm{SD}$ ).

\begin{tabular}{|c|c|c|}
\hline Phytoplankton $\left(10^{3}\right.$ cells. $\left.\mathrm{L}^{-1}\right)$ & Non-Red & Red \\
\hline Euglenophyta & $0.38 \pm 0.14^{\mathrm{b}}$ & $2.26 \pm 0.36^{\mathrm{a}}$ \\
\hline Euglena sanguinea & $0.20 \pm 0.05^{\mathrm{b}}$ & $1.61 \pm 0.42^{\mathrm{a}}$ \\
\hline Euglena acus & $0.10 \pm 0.06^{\mathrm{b}}$ & $0.25 \pm 0.08^{\mathrm{a}}$ \\
\hline Trachelomonas & $0.05 \pm 0.03^{\mathrm{b}}$ & $0.27 \pm 0.09^{\mathrm{a}}$ \\
\hline Phacus & $0.04 \pm 0.03^{\mathrm{b}}$ & $0.13 \pm 0.02^{\mathrm{a}}$ \\
\hline Chlorophyta & $2.38 \pm 0.51^{\mathrm{a}}$ & $0.66 \pm 0.05^{\mathrm{b}}$ \\
\hline Cyanophyta & $0.41 \pm 0.14^{\mathrm{a}}$ & $0.45 \pm 0.15^{\mathrm{a}}$ \\
\hline Bacillariophyta & $0.37 \pm 0.04^{\mathrm{a}}$ & $0.38 \pm 0.06^{\mathrm{a}}$ \\
\hline Total & $3.54 \pm 0.74^{\mathrm{a}}$ & $3.74 \pm 0.54^{\mathrm{a}}$ \\
\hline \multicolumn{3}{|l|}{ Zooplankton $\left(10^{3}\right.$ cells. $\left.\mathrm{L}^{-1}\right)$} \\
\hline Rotifera & $0.18 \pm 0.02 b$ & $0.25 \pm 0.04 \mathrm{a}$ \\
\hline Cladocera & $0.37 \pm 0.02 \mathrm{a}$ & $0.30 \pm 0.07 \mathrm{a}$ \\
\hline Copepoda & $0.26 \pm 0.04 \mathrm{a}$ & $0.32 \pm 0.07 \mathrm{a}$ \\
\hline Total & $0.81 \pm 0.06 a$ & $0.87 \pm 0.16 \mathrm{a}$ \\
\hline
\end{tabular}

Mean values with different superscript letters in the same row are significantly different $(\mathrm{P}<0.05)$.

Table 5. Gross margin analysis for non-red pond and red pond with algal bloom (NPR ha'-1) in 120 days.

\begin{tabular}{|l|c|c|}
\hline \multirow{2}{*}{ Variable cost } & \multicolumn{2}{c|}{ Treatments } \\
\cline { 2 - 3 } & Non-red & Red pond \\
\hline Carp Seed & $20000 \pm 0.0^{\mathrm{a}}$ & $20000 \pm 0.0^{\mathrm{a}}$ \\
\hline DAP & $25200 \pm 0.0$ & 0.0 \\
\hline Urea & $11844 \pm 0.0$ & 0.0 \\
\hline Total fertilizer & $37044 \pm 0.0$ & $195784 \pm 7648^{\mathrm{a}}$ \\
\hline Feed & $176134 \pm 1047^{\mathrm{a}}$ & $215784 \pm 7648^{\mathrm{a}}$ \\
\hline Total variable cost & $233178 \pm 10463^{\mathrm{a}}$ & \\
\hline Return & & \\
\hline
\end{tabular}




\begin{tabular}{|l|c|c|}
\hline Fish production $\left(\mathrm{kg} \mathrm{ha}^{-1}\right)$ & $2238.1 \pm 188.4^{\mathrm{a}}$ & $1999.9 \pm 464.6^{\mathrm{a}}$ \\
\hline Gross return $\left.(\mathrm{NPR} \mathrm{ha})^{-1}\right)$ & $604269 \pm 50866^{\mathrm{a}}$ & $539961 \pm 12546^{\mathrm{a}}$ \\
\hline Gross margin $\left(\mathrm{NPR} \mathrm{ha}^{-1}\right)$ & $371092 \pm 59002^{\mathrm{a}}$ & $324177 \pm 117832^{\mathrm{a}}$ \\
\hline B/C ratio & $2.6 \pm 0.3^{\mathrm{a}}$ & $2.5 \pm 0.5^{\mathrm{a}}$ \\
\hline
\end{tabular}

Mean values with different superscript letters in the same row are significantly different $(\mathrm{P}<0.05)$.

of red algae. This finding is in conformity with the previous report (Mandal et al., 2016; Boyd and Tucker, 2014).

\section{Plankton}

Euglenophytes abundance in red pond was significantly higher $\left(2260 \pm 360\right.$ cells $\left.\mathrm{L}^{-1}\right)$ than non-red pond $\left(380 \pm 140\right.$ cells $\left.\mathrm{L}^{-1}\right)$, while chlorophytes showed antagonistic in nature with reference to euglenophytes and it was significantly higher in non-red pond $(2380 \pm 510$ cells $\left.\mathrm{L}^{-1}\right)$ than red pond $\left(660 \pm 50\right.$ cells $\left.\mathrm{L}^{-1}\right)$ (Table 4). Cyanophytes and Bacillariophytes were not significantly different $(\mathrm{P}>0.05)$ between red and non-red ponds. In red ponds, Euglena sanguinea was more than $71 \%$ of total Euglenophyte population. Total population of phytoplankton did not differ $(\mathrm{P}>0.05)$ between non-red and red ponds. Rotifer was found significantly higher $(\mathrm{P}<0.05)$ in red pond $\left(250 \pm 40\right.$ cells $\left.\mathrm{L}^{-1}\right)$ than non-red pond $\left(180 \pm 20\right.$ cells $\left.\mathrm{L}^{-1}\right)$ but Cladocera and copepod did not vary $(\mathrm{P}>0.05)$ between red and non-red ponds. Total population of zooplankton was not found significantly different $(\mathrm{P}>0.05)$ between red and non-red ponds.

In the red ponds, high nutrients such as TP and TN were found which is quite similar to Xavier et al. (1991) and Rahman et al. (2007). Population of euglenophytes in red pond was found to be higher compared to chlorophytes, bacillariophytes and cyanophytes in nutrient rich water bodies (Costa and Garrido, 2004; Lopez et al. 2008; Costa, 2014). Red pond also favors the growth and density of rotifer, so, the density was higher in red ponds than non-red ponds which probably because euglenophytes served as food to them (Kozak et al., 2015).

\section{Gross margin analysis}

Gross margin analysis showed that both treatments were profitable (Table 5). Gross margin was comparatively higher in the non-red pond $\left(371092 \pm 59003 \mathrm{NPR} \mathrm{ha}^{-1}\right)$ than red pond $\left(324177 \pm 117832\right.$ NPR ha $\left.{ }^{-1}\right)$. Similarly benefitcost ratio was not significantly different $(\mathrm{P}>0.05)$ but comparatively higher in the non-red pond $(2.6 \pm 0.3)$ than that of the red pond $(2.5 \pm 0.5)$.
Gross margin analysis showed that both treatments were profitable due to higher fish yields. Gross margin and benefit-cost ratio were also not different between two treatments because fish yield was same.

\section{Conclusion}

Present study showed that red pond caused by blooming of Euglena sanguinea had no effect on overall production of carp and gross margin. So, red algal bloom in ponds is not a threat to fish farmers. It is a good relief to carp farmers in Nepal who grows carp in a semi-intensive way on natural foods such as phytoplankton and zooplankton and supplemental feeds. In Nepal, over $90 \%$ farmers grow carp semi-intensively in fertilized ponds. Intense red bloom might increase nitrite and decrease DO that may cause fish mortality.

\section{Acknowledgements}

We would like to thank Fisheries Program, Agriculture and Forestry University (AFU) for providing lab facilities. We also thank to staff of Aquaculture Farm for kind help in fish sampling. This research was funded by Aqua Fish Innovation Lab, USAID through Agriculture and Forestry University, Rampur, Chitwan, Nepal/

\section{References}

APHA. 2012. Standard methods for the examination of water and waste water (22 $\left.2^{\text {nd }} \mathrm{Ed}.\right)$. American Public Health Association, Washington.

Boyd, C.E. and C.S. Tucker 2014. Hand book for water quality. Alabama Agriculture Experiment Station, Auburn University, Alabama. pp 237-251.

Ciji, A., N.P. Sahu, A.K. Pal, M.S. Akhtar, V. Tincy, P. Mishal and P. Das 2014. Effect of dietary vitamin $\mathrm{E}$ and nitrite exposure on growth and metabolic variables of Labeo rohita juveniles. National Academy Science Letter 37(2): 123-129.

Costa, P.R. 2014. Impact and effects of paralytic shellfish poisoning toxins derived from harmful algal blooms to marine fish. John Wiley and Sons Ltd.

Costa, P.R. and S. Garrido 2004. Domoic acid accumulation in the sardine Sardina pilchardus and its relationship to Pseudonitzschia diatom 
ingestion. Marine Ecology Progress Series 284: 261-268.

Dhanapathi, M.V.S.S.S. 2000. Taxonomic notes on the Rotifera. Indian Association of Aquatic Biologist, Hyderabad, India. 178p.

DoFD, 2016. Annual Progress Report. Govt. of Nepal, Directorate of Fisheries Development. Balaju, Kathmandu, Nepal.

Edmondson, W.T. 1959. Fresh water biology $\left(2^{\text {nd }}\right.$ Ed.). Edward and Hipple (Eds.), John Willy and Sons Inc, New York. pp 95-189.

Guiry, M.D. and G.M. Guiry 2016. Algae Base. World-wide electronic publication, National. University of Ireland, Galway. http://www.algaebase.org.

Kozak, A., R. Gołdyn and R. Dondajewska 2015. Phytoplankton composition and abundance in restored Maltański Reservoir under the influence of physico-chemical variables and zooplankton grazing pressure. PLOS ONE 10(4): e0124738. DOI: 10.1371/journal.pone.0124738

Lopez, C.B., E.B. Jewett, Q. Dortch, B.T. Walton and H.K. Hudnell 2008. Scientific assessment of freshwater harmful algal blooms. Interagency Working Group on Harmful Algal Blooms, Hypoxia, and Human Health of the Joint Subcommittee on Ocean Science and Technology, Washington. DC. 65p.

Mandal, R.B., S. Rai, M.K. Shrestha, D.K. Jha, N.P. Pandit and S.K. Rai 2016. Water quality and red bloom algae of fish ponds in three different regions of Nepal. Our Nature 14(1): 71-77.

Ohi, E.P.A. 2013. Seeing red: Emerging harmful algal blooms. Newsletter for Ohio's Public Drinking Water Systems. Spigot News summer 2013 Page 2.

Pennak, R.W. 1978. Freshwater invertebrate of United States ( $2^{\text {nd }}$ Ed.). John Wiley and Son, New York. 303p.

Prescott, G.W. 1951. Algae of the western great lakes area. WM.C. Brown Publishers, Dubuque, Iowa, 977p.

Rahman, M.M. and S. Khan 2007. Noxious euglenop- phytes bloom in fertilized fish ponds. Bangladesh J. Fish. Res. 11(1): 7-18.

Rahman, M.M., M.A. Jewell, S.S. Khan and M.M. Haque 2007. Study of euglenophytes bloom and its impact on fish growth in Bangladesh. Algae 22(3): 185-192.

Rai, S., Y. Yi1, Md. A. Wahab, A.N. Bart and J.S. Diana 2010. Comparison of the growth and production of Carps in polyculture ponds with dupplemental feed using rice straw and Kanchi as substrates. Our Nature 8: 92-105.

Rai, S.K. and R.K. Rai 2007. Some euglenophycean algae from Biratnagar, Nepal. Our Nature 5: 60-6.

Reddy, Y.R. 1994. Copepod, cladocera, diaptomidous guide to the identification of micro invertebrate of the continental water of the world. SPB Publisher, The Hague, Netherland.

Rehman, S.U. 1998. A red bloom of Euglena shafiqii, a new species in Dal Lake, Srinagar, Kashmir. Water Air and Soil Pollution 108: 69-82.

Rodgers, J.H. 2008. Algal toxins in pond aquaculture. SRAC Publication No. 4605: 1-8.

Shang, Y.C. 1990. Aquaculture economics analysis: An introduction. World Aquaculture Society, Baton Rouge, Louisiana. 211p.

Wild, A., J. Lee-Kaden, H. Gimmler and W. Simonis 1995. Phycological and hydro-chemical investigations of two polytrophic ponds near to an urban development. Limnology 25: 229-236.

Xavier, M.B., C.S.R. Mainardes-Pinto and M. Takino 1991. Euglena sanguinea Ehrenberg bloom in a fish-breeding tank (Pindamonhangaba, Sao Paulo, Brazil). Archive fur Hydrobiology. Supplement band, Algological Studied 62: 133-142.

Zimba, P.V., M. Rowan and R.E. Triemer 2004. Identification of euglenoid algae that produce ichthyotoxin(s). Journal of Fish Diseases 27: 115117.

Zimba, P.V., P.D. Moeller, K. Beauchesne, H.E. Lane and R.E. Triemer 2010. Identification of euglenophycin- A toxin found in certain euglenoids. Toxicon 55(1): 100-104. DOI: 10.1016/j.toxicon.2009.07.004. 